\title{
Solar granulation properties derived from three different time series
}

\author{
D. Del Moro ${ }^{1,2}$ \\ 1 Dipartimento di Fisica, Università di Roma "Tor Vergata", 00133 Roma, Italy \\ e-mail: delmoro@roma2.infn.it \\ 2 CNR - IFSI, 00133 Roma, Italy
}

Received 18 March 2004 / Accepted 7 July 2004

\begin{abstract}
We need automatic pattern recognition algorithms to extract a large statistical sample of granules from high spatial and temporal resolution images series of photospheric fields.

In this paper, we present the new Two-level Structure Tracking (TST) algorithm, based on a two-level representation of granulation, which allows us to monitor the characteristics of identified photospheric structures during their duration. TST is also able to retrieve horizontal velocity maps from measured granule displacements. Direct comparison of the results from different works describing granular evolution is often not possible as discrepancies stemming from the use of different procedures or from different data cannot be distinguished.

Here, three different solar granulation broadband time series, acquired at THEMIS in July 1999, at NSO-DST in October 1996, and at SVST in June 1995, with different spatial and temporal resolutions, are coherently analyzed via the same TST procedure, allowing direct comparison of the results.

Among the obtained results, we confirm the dynamical heterogeneity of photospheric small scale structures pointed out by granular lifetime histograms, characterized by a stretched exponential function.

Furthermore, by monitoring the breaking-up of a granule and the movements of its neighbours and of its fragments, we found evidence of how an exploding granule may produce a divergence signal on spatial and temporal mesogranular scales.
\end{abstract}

Key words. methods: numerical - Sun: photosphere - methods: data analysis

\section{Introduction}

Convective instabilities present in the Sun external layers produce matter flows which are responsible for the appearance of intensity patterns, with different spatial scales, on the solar surface. The physical properties of convection (Rast 2003), particularly of small scale convective features (granules), have been investigated using both computer simulations (Nordlund 1984; Stein \& Nordlund 1998; Steffen et al. 1989; Gadun et al. 2000) and high resolution spatial and spectral observations (see references in Koschincky et al. 1999 and Rast 2003). The study of granular advection properties and lifetimes has been carried out by several authors (see references in Spruit et al. 1990 and Berrilli 2003) and represents an important aspect of the more general investigation on photospheric plasma convective characteristics. The observation of surface flows and the determination of granule mean lifetimes can be compared with the results of convective theories and simulations.

In order to trace horizontal flows on the solar photosphere, the small-scale convective structures, granules, may be used. With the assumption that granule motion is mainly driven by plasma flows, it is possible to employ two approaches to infer horizontal velocity fields: local correlation or structure tracking techniques.
An interesting analysis of the reliability of horizontal velocity fields and divergence signals derived from granule tracking has been carried out by Roudier et al. (1999) and by Rieutord et al. (2001), concluding that granules tend to be good tracers for spatial scales greater than $2.5 \mathrm{Mm}$ and for temporal scales longer than $30 \mathrm{~min}$, while for smaller scales the derived plasma flow is spoilt by the granules own velocity field. This study therefore provides strong constraints for flow field extraction, in particular implies rejecting as trackers all the granules whose structure deformation rate is excessive.

In recent years, structure tracking methods have been used by Hirzberger et al. (1997, 1999a,b) to study the evolution of individual solar granules and by Rieutord et al. (2000) to investigate the mesogranulation properties.

When dealing with previous works, special care must be taken in the evaluation of how different tracking procedures and/or different spatial and temporal sampling intervals may affect the results. In particular, different methods of image segmentation and granule definition may explain the discrepancies between different published granule sizes distributions (Roudier \& Muller 1987; Title et al. 1989; Brandt et al. 1991; Schrijver et al. 1997; Bovelet \& Wiehr 2001; Berrilli et al. 2002). Again, low temporal resolution and different conditions for granule death may alter lifetime distributions; for example, 
Hirzberger et al. (1999a) derive a mean lifetime of $\sim 5 / 6$ min, while Roudier et al. (1999) derive a mean lifetime of $\sim 2$ min.

Also the dependence of several granule properties (primarily size) on their duration has been investigated by some authors (Title et al. 1989; Hirzberger et al. 1999a), in particular, Hirzberger et al. (1999a) derive distributions in good agreement with numerical simulations of convective structures (Ploner et al. 1998; Gadun et al. 2000).

Regrettably, comparing those results may be a very frustrating task as it is very difficult to discern whether the discrepancies arise from differences in the procedures or the data. To allow direct comparison, three solar granulation broadband time series acquired from different telescopes and with different spatial and temporal resolutions are coherently analyzed via the same procedure that simplifies granules in Two-level Structures and tracks them.

\section{Observations and image pre-processing}

The first image sequence (hereafter THEMIS99) analyzed in this work, was acquired at the THEMIS telescope (Observatorio del Teide, Tenerife, Spain) in IPM (Italian Panoramic Monochromator) observing mode on July 1, 1999 (from 7:21 UT to 8:24 UT). It is made up of 608 broad-band (central wavelength $538.0 \mathrm{~nm}$, band-pass $4 \mathrm{~nm}$ ) $30^{\prime \prime} \times 30^{\prime \prime}$ $(223 \times 223$ pixels $)$ images with $0.04 \mathrm{~s}$ exposure time. After the standard corrections (Flat-Field and Dark Current) and Recentring (rigid alignment alone) the acoustic modes pattern was removed by a $k_{h}-\omega$ filter, with a cut-off velocity of $6 \mathrm{~km} \mathrm{~s}^{-1}$, and a Wiener filter was applied to the images.

For a more detailed description of this dataset refer to Berrilli et al. (2001).

The second image sequence (hereafter NSO96) was acquired at the NSO Dunn Solar Telescope (Sacramento Peak, Sunspot, New Mexico) on October 16, 1996 (from 14:30 UT to $16: 30 \mathrm{UT}$ ). This broadband (central wavelength $550.0 \mathrm{~nm}$, band-pass $5 \mathrm{~nm}$ ) sequence is made of 512 images with field of view of $31.5^{\prime \prime} \times 31.5^{\prime \prime},(256 \times 256$ pixels $)$, each taken with an exposure time of $0.008 \mathrm{~s}$. After the standard corrections, this series was treated with a destretching algorithm and the acoustic modes pattern was removed by $k_{h}-\omega$ filtering with a cut-off velocity of $4.7 \mathrm{~km} \mathrm{~s}^{-1}$.

For a more detailed description of this dataset refer to Cauzzi et al. (1998).

The third time series (hereafter SVST95) was acquired at the Swedish Vacuum Solar Telescope (Roque de los Muchachos, La Palma, Spain) on June 30, 1995 (from 10:34 UT to 12:50 UT). The series is made up of 220 broadband (central wavelength $525.7 \mathrm{~nm}$, band-pass $3 \mathrm{~nm}$ ) images with field of view of $18^{\prime \prime} \times 18^{\prime \prime},(290 \times 290$ pixels $)$, each taken with an exposure time of $0.011 \mathrm{~s}$. After the standard corrections this series was sub-pixel re-centred, destretched and treated with a $k_{h}-\omega$ (cut-off velocity $4 \mathrm{~km} \mathrm{~s}^{-1}$ ) and a restoration filter. For a more detailed description of this dataset refer to Sobotka et al. (1999).

All the three series show images of quiet granulation at the disk centre.

\section{TST: Two-level structure tracking procedure}

We developed and applied to the image series an automatic procedure of recognition and tracking, in order to objectively describe morphological and dynamic properties of solar features. The Two-level Structure Tracking (TST henceforth) procedure recognizes structures in single images, to derive morphological and topological information on such structures, and, by using image time series, to trace structures in time, in order to investigate their time properties and reconstruct horizontal velocity fields.

The TST procedure can be divided into four main steps:
(a) segmentation;
(b) labelling;
(c) tracking;
(d) velocity field reconstruction.

Step a:

The segmentation task breaks up the pre-processed image into structures by assigning each pixel to a coherent structure via a statistic evaluation of its surroundings (Berrilli et al. 1998). This is done using a threshold which is evaluated on a moving box of $L \times L$ centred on the pixel under analysis, where $L$ is comparable to structure linear dimensions. The threshold function is:

$T\left(x, y, S_{x y}\right)=\langle I(x, y)\rangle+\xi \cdot \operatorname{rms}(x, y)$

that is the mean of the sub-image plus $\xi$ times its standard deviation.

The optimal parameter values for the segmentation are the $\left[L_{0}, \xi_{0}\right]$ such that

$\left.\frac{\mathrm{d}^{2} N(L, \xi)}{\mathrm{d} L \mathrm{~d} \xi}\right|_{\left[L_{0}, \xi_{0}\right]}=\min \left(\frac{\mathrm{d}^{2} N(L, \xi)}{\mathrm{d} L \mathrm{~d} \xi}\right)$

for $0.0<\xi \leq 2.0$ and $0.0^{\prime \prime}<L \leq 3.0^{\prime \prime}$, where $N(L, \xi)$ is the identified structures number (regardless of their size). The values we retrieved for the segmentation of the THEMIS99 and NSO96 time series are $L=1.5^{\prime \prime}$ and $\xi=0.8$, while for SVST95 we found $L=1.5^{\prime \prime}$ and $\xi=0.5$.

The output of this step is a two-level, or black and white, version of the image (Fig. 1).

Step $b$ :

After an image has been segmented into structures, we label the structures with a unique index. We retain as granules the structures whose areas range from 0.1 to $3 \mathrm{Mm}^{2}$, with a "regular" geometry: the morphological describer $M=$ Perimeter $^{2} /(4 \pi \times$ Area $)$, being 1 for a circle, has proved to be a good appraiser for the regularity of structures. If the procedure is used to reconstruct horizontal velocity fields, we found it reasonable to keep structures whose $M$ is less than a fixed value, in order to discard structures whose deformations can introduce spurious barycentre motion. The value $M=4$ represents a good compromise to retain "regular" convective features and discard structures with complex morphology.

After this selection, the procedure extracts and stores geometric and intensity describers of identified structures in 


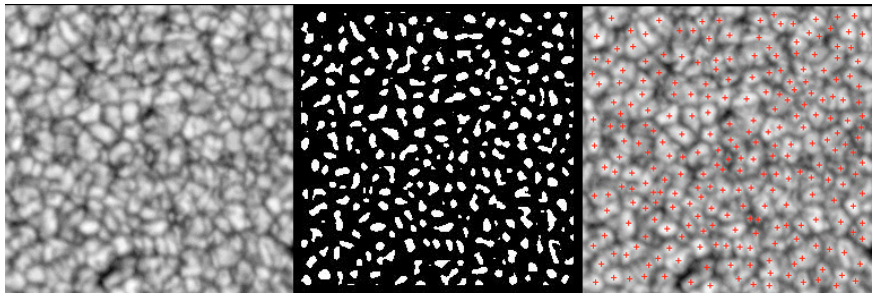

Fig. 1. Left: pre-processed NSO96 image. Center: same image as a two-level image. Right: same image with the positions of the barycentres of recognized granules superimposed.

a database. The outputs of steps $a$ and $b$ relative to a sample image of NSO96 series are shown in Fig. 1.

Step c:

The structure tracking is accomplished using both the information collected by step $b$ and the two-level granulation images produced by step $a$. In detail, starting from the first image of the temporal series, all stored structures are selected and tracked forward in time. For each structure a circular region, centred in its barycentre and with a $5^{\prime \prime}$ radius, is defined. All the structures of the succeeding image, whose barycentres are inside this region, are compared in shape with the original structure. The one whose shape closest matches, within a maximum allowed deformation value (10\%), is retained and considered as the evolution of the original structure, otherwise the lineage ends. Once all the structures of this image have been processed, the TST proceeds to the next, tracking all the structures which have not been identified as evolutions of previous images granules.

With these rules a granular lineage ends as the structure becomes too small (fading) or too large (merging), or it warps too much or too fast (merging or exploding events). In this way we produced a database whose entries are ordered by structure number, containing the related structure characteristic at a fixed time. This process is repeated until the last structure in the last image is reached.

We do not reject structures that appear on either the first or last image, which for each time series are less than $10 \%$. Consequently, the distributions will be slightly biased toward short-lived granule characteristics. We chose to include these structures in all the distributions because removing them would bias the results even more toward short-lived granule characteristics, for longer-lived granules have a greater probability of appearing on either the first or last image in the time series than the granules with shorter lifetimes.

\section{Step d:}

Horizontal velocity fields are derived using the intensity barycentre motion of identified structures. In order to remove seeing-induced displacements, we compute the average barycentre position each $30 \mathrm{~s}$. The displacements of averaged barycentres are used to derive a sparse sampled array of velocity vectors using a $60 \mathrm{~s}$ time window. Thus, we obtain a fully independent velocity field each $90 \mathrm{~s}$, which is associated with the image in the middle of the time window. The sparse array of velocity vectors is transformed into a regularly gridded one by applying a Delaunay triangulation (Lancaster \& Salkauskas 1986; Alfeld 1989).

To test its reliability, the whole TST procedure has been applied to simulated granulation time series, successfully retrieving the synthetic velocity field.

\subsection{TST versus CST: A comparison}

The retrieving of horizontal velocity fields from granule motion is a topic which has been tackled by some authors in the past. In particular, two main techniques have been developed: LCT (Local Correlation Tracking) by November \& Simon (1988) and FT (Feature Tracking) by Strous (1995). In particular Roudier et al. (1999) have developed an improved version of FT, namely the CST (Coherent Structure Tracking) algorithm. We present here a brief comparison of TST and CST characteristics. Both algorithms retrieve the horizontal velocities by tracking the displacements of "coherent features" (granules), identified by their centre (i.e. without intensity information) in the CST case, or by their intensity barycentre in the TST case. The structures are identified by different methods: CST structures are recognized by searching for the zeros of the second derivative of the intensity field and then by applying both a Gaussian smoothing window to remove noise and an Opening operator in order to remove isthmuses and protrusions. TST structures are instead retrieved by recognition of clusters of pixels which are brighter than their surroundings. Both methods avoid problems caused by large scale intensity variations and efficiently retrieve segmented granular patterns. A common element of both procedures is the evaluation of the regularity of segmented structures by a morphological describer, in order to discriminate true motion from spurious barycentre movement due to structure deformation. This is a crucial point for both procedures: for TST, because tolerating too much structure deformation would forbid discriminating spurious barycentre movement from true structure motion, much more for CST, which relies on minor morphologic changes of structures from one frame to the other. In fact, CST reconstructs granule lineages by searching the surroundings of the barycentre position between successive images and hence would be sensitive to splitting and merging events. TST, instead, while still requiring barycentre proximity in consecutive frames, validates the detection by comparing the structure shapes. Moreover, in order to cope with seeing-induced motion, barycentre positions are averaged over $30 \mathrm{~s}$ before computing their displacements. However, the most crucial difference is in the field reconstruction, as the transformation of a randomly sampled field to a regular grid can introduce undesirable elements originating from the interpolation algorithm itself. In order to retrieve the uniformly sampled field, the CST algorithm computes the average of the values in an established neighbourhood, therefore not exploiting the distance information. As an alternative solution, the field is reconstructed by using LCT, with a Gaussian averaging window of about half the granules' mean size. The TST procedure instead approximates grid values by Delaunay linear interpolation, thus weighting the contribution of the sparsely-sampled array points by their distances 


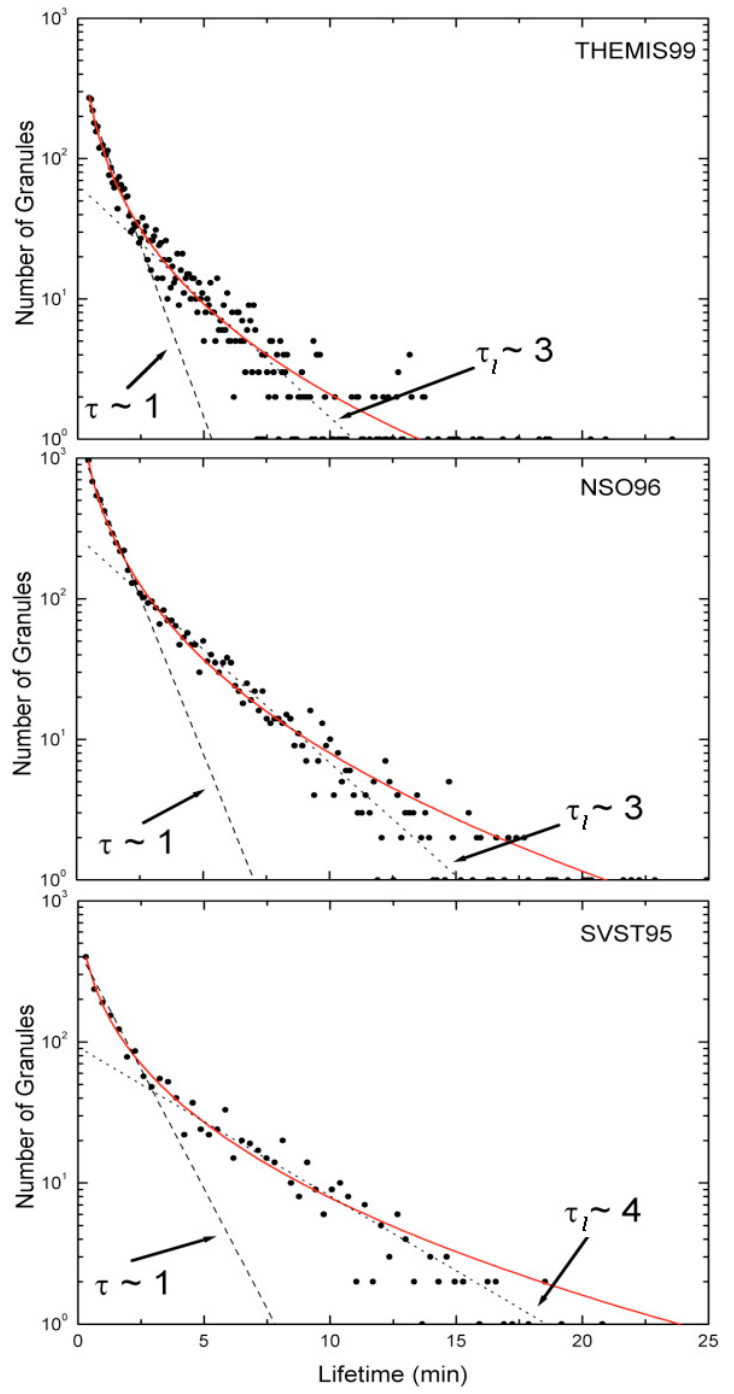

Fig. 2. Histograms of granule lifetime from THEMIS99, NSO96, SVST95 series. Dotted and dashed black lines are exponential decay fits for the whole distribution (deriving $\tau \sim 1$ ) and for points with $t \geq 5$ (deriving $\tau_{l} \sim 3 / 4$ ), respectively. The continuous lines are stretched exponential fits to the data.

from new grid junctions; moreover, the accuracy of the result can be estimated as a function of the distance from sampling points.

\section{Results and discussion}

When applied to the datasets described in Sect. 2, the TST procedure tracked 4173 lineages in the THEMIS99 time series (608 images), 6940 in the NSO96 time series (512 images) and 1713 in the SVST95 time series (220 images).

\subsection{Granular lifetime distributions}

The temporal behaviour of recognized granules can be investigated using their lineages. The lifetimes can be obtained measuring the time elapsing between their birth and death, deriving the distribution functions reported in Fig. 2.
Table 1. Fit parameters: for each dataset we report the values of the significant parameters of the fits: $\tau$ is the decay time for an exponential fit taking into account all the points of the lifetime histogram; $\tau_{l}$ is the decay time for an exponential fit taking into account only lifetimes $\geq 5 \min ; \alpha$ and $\tau_{\mathrm{se}}$ are the relaxation parameter and the decay time for the stretched exponential fit, respectively.

\begin{tabular}{lllll}
\hline \hline Timeseries & $\tau(\min )$ & $\tau_{l}(\min )$ & $\alpha$ & $\tau_{\text {se }}(\min )$ \\
\hline THEMIS99 & $0.9 \pm 0.02$ & $2.6 \pm 0.1$ & $0.27 \pm 0.03$ & $0.003 \pm 0.001$ \\
NSO96 & $1.0 \pm 0.02$ & $2.7 \pm 0.1$ & $0.33 \pm 0.02$ & $0.024 \pm 0.008$ \\
SVST95 & $1.3 \pm 0.05$ & $4.1 \pm 0.2$ & $0.37 \pm 0.02$ & $0.10 \pm 0.03$ \\
\hline
\end{tabular}

Our stringent birth and death conditions, set in order to retain only very coherent structures, define a different "granule" to that used in previous works. As in the case of Roudier et al. (1999), the obtained lifetime is a coherence time scale rather than the proper structure duration.

Following Title et al. (1989) and Roudier et al. (1999) we chose to fit the histograms with a decaying exponential law

$N(t)=N(0) \mathrm{e}^{-t / \tau}$

(Alissandrakis et al. 1987), reported as black dotted and dashed lines in Fig. 2, to estimate mean lifetimes, as for a decaying exponential distribution $\bar{t}=\tau$ (Hirzberger et al. 1999a).

Regrettably, this function seems unable to represent the reported lifetime distributions, either for short or for long granule duration. In fact, as can be seen in Fig. 2, the decay law of lifetimes seems to be at least twofold, indicating different regimes for $t<\sim 2.5$ or $t>\sim 2.5 \mathrm{~min}$. This double exponential behaviour could account for the presence of at least two different granular populations: a bulk with a very short mean lifetime $(\bar{t} \sim 1 \mathrm{~min})$ and a minority with a longer mean lifetime $(\bar{t} \sim 3 / 4 \mathrm{~min})$.

A comparable distribution is reported by Brandt et al. (1991), and a closer look at the granule lifetime distributions in Title et al. (1989) reveals a similar excess of short lived granules in four out of six reported lifetime histograms.

In the present work, we observe that granule lifetime histograms may be properly approximated by a stretched exponential (Williams \& Watts 1970) function (continuous line in Fig. 2):

$N(t)=N(0) \mathrm{e}^{-\left(t / \tau_{\mathrm{se}}\right)^{\alpha}}$.

The stretched exponential seems to be a universal function obeyed by slow relaxations, describing systems driven out of equilibrium. The parameters $\tau_{\text {se }}$ and $\alpha$ are a decay time (although not directly related to granule mean lifetime) and a dispersive factor, respectively. Values of $\alpha<1$ correspond to the existence of a broad distribution of lifetimes which describe the elementary relaxation process. In this framework, the lifetime histograms suggest that we cannot define a typical lifetime for granules, which instead show a continuous distribution of decay times. Here, we report the exponential fits and the stretched exponential fit parameters for the three datasets and briefly discuss the results. 
The datasets essentially agree for $\tau, \tau_{l}$ and $\alpha$ values, but there is significant divergence in the $\tau_{\mathrm{se}}$ values and, to some extent, for the $\tau_{l}$ values. The key difference to explain the disagreement in the $\tau_{l}$ values is most likely the higher spatial resolution of the SVST95 dataset, which facilitates retrieving longer lasting structure; instead, the small differences between NSO96 and THEMIS99 are probably due to the different reduction methods. In particular, the re-centring process applied to the THEMIS99 dataset is able to correct for seeing-induced tip/tilt in the central part of the Fov, leaving tip/tilt in different isoplanatic patches uncorrected. This leads to spurious motion of granules in the periphery of the image that may cause the splitting of a single granular lineage in two, thus creating two short-lasting granules from a long-lasting one, and populating the short-lasting part of the histogram at the expense of the long-lasting part.

The spread of the $\tau_{\text {se }}$ values over two order of magnitude can be explained by the extreme sensitivity of this parameter to the histogram shape, implying a rather different ratio of short to long lived granules in the three datasets. In the stretched exponential representation, the $\tau_{\mathrm{se}}$ values do not correspond to mean lifetimes, as in the single exponential function, because a typical lifetime cannot be defined.

The substantial concord for the $\alpha$ value is more interesting, as it is indicative of the existence of a broad lifetime distribution, therefore confirming the conclusions of Berrilli et al. (2002), inferring that the dynamics of the low photosphere "resembles the behaviour of a complex out-of-equilibrium system characterized by a dynamical heterogeneity".

\subsection{Granular area distribution}

Using the TST procedure we are able to compute the average area during its lifetime for each tracked granule. This area refers to identified structure and not to the granular cell. Moreover, the structure area depends on the choice of the segmentation threshold. However, by using an i-MAT algorithm (Berrilli et al. 1998), it is possible to evaluate the dimensions of the cell associated with the structure. As we need such relationship only for statistical use, not for a one-to-one representation, we computed the structure dimension vs. cell dimension relation for a subset of the structures instead of for the whole collection. The structure dimension vs. cell dimension is plotted in Fig. 3. The relationship is linear, with a cell-structure ratio of $3.2 \pm 0.1$. We used this multiplying factor to rescale the axis of the computed structure plots, thus obtaining a histogram of granule number versus their time-averaged structure or cell area, shown in Fig. 4.

The three series concur in retrieving an increase of the number of granules towards smaller ones until 1.0/1.5 $\mathrm{Mm}^{2}$ cell area, followed by a plateau until the lower limit cut-off (due to our forced rejection of structures whose area is smaller than $0.1 \mathrm{Mm}^{2}$ (cf. 3)) is reached. In spite of the $3 \mathrm{Mm}^{2}$ forced area limit (cf. 3), the histograms substantially agree in the upper limit cut-off, showing that virtually no granule has an average structure area greater than $1 \mathrm{Mm}^{2}$ (and hence average cell diameter $\sim 2.0 \mathrm{Mm}$ ). It is interesting to compare these

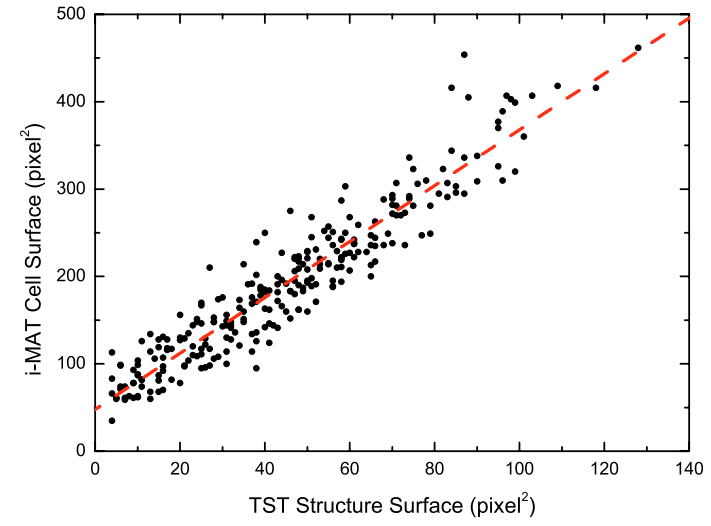

Fig. 3. Area of i-MAT cell vs. associated TST structure area extracted from the sample image in Fig. 1.

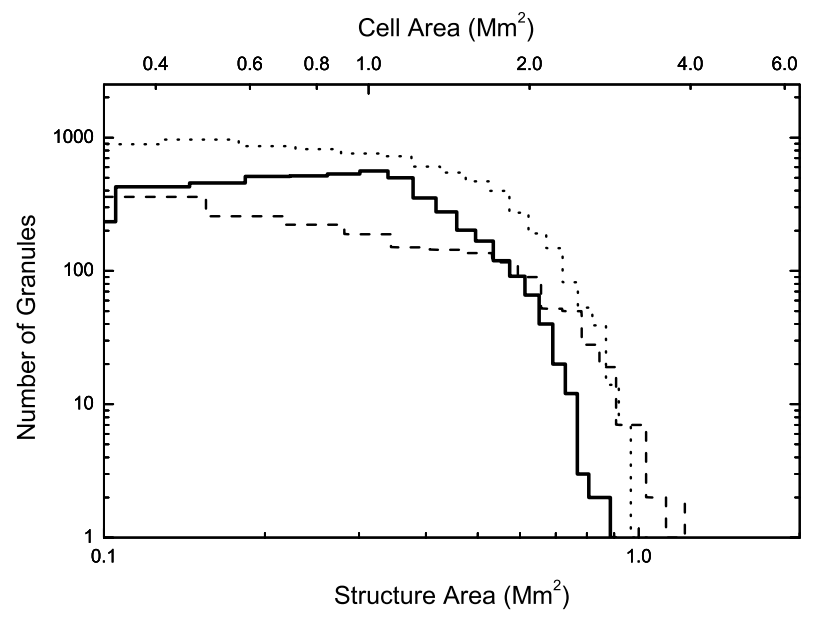

Fig. 4. Histograms of granule mean area during lifetime from THEMIS99 (solid), NSO96 (dotted) and SVST95 (dashed) series.

histograms obtained using areas of granules averaged over their lifetime to similar histograms, such as those reported by Roudier \& Muller (1987), Title et al. (1989), Hirzberger et al. (1997), obtained from "instantaneous" granule areas, i.e. from single images. Taking into account the different granule definitions, our findings are compatible and confirm the previous results quoted, except for the different large scale cut-off of Roudier \& Muller (1987) and Hirzberger et al. (1997) cell area histograms around 3.0 and $5.0 \mathrm{Mm}^{2}$, respectively. This discrepancy is likely due to the time-averaging of the granule areas we performed in order to retrieve the mean area of a granule during its lifetime.

\subsection{Granular area and intensity versus lifetime}

The dependence of the average area and intensity of the structure on the lifetime has been investigated. Area versus lifetime plots in Fig. 5 refer to the mean or maximum area of the structure during its lifetime, i.e. for a given time bin we have retrieved the granule lineages with corresponding durations, computed the average and maximum area throughout their lifetime, and then calculated the mean of these values in the same time bin. Intensity versus lifetime plots in Fig. 6 refer instead to the mean or maximum intensity (normalized to proper series 


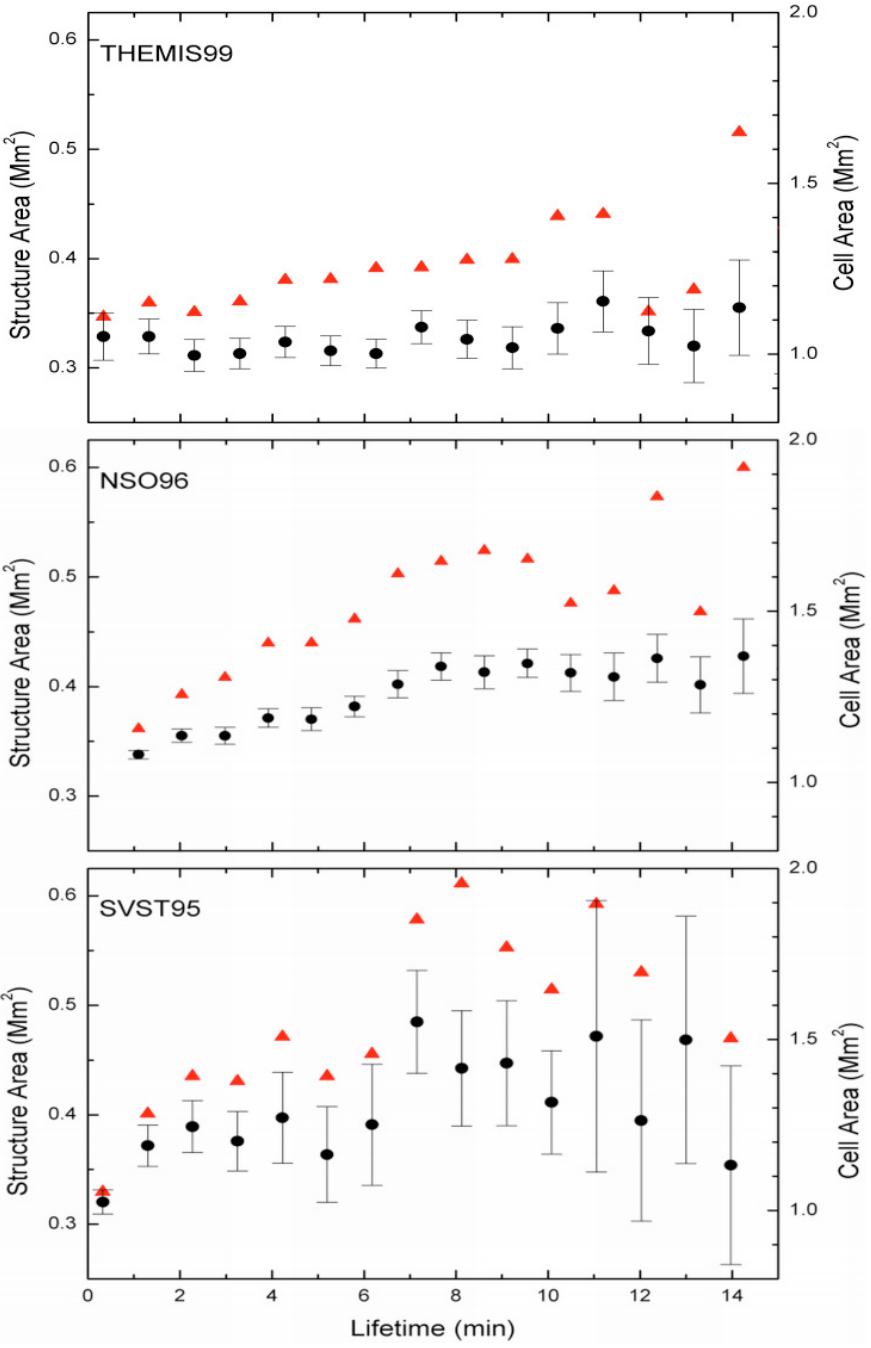

Fig. 5. Plot of granule mean (black filled circles with error bars) and maximum (grey triangles) area during lifetime versus their lifetime. Maximum area error bars, comparable to mean area error bars, are omitted for clarity.

intensity scale maximum) inside the tracked structure during its lifetime; the histograms have been obtained in the same way as area histograms. Lifetimes longer than 15 min have been excluded from plots due to their very poor statistics.

For lifetimes shorter than 8 min, granular mean and maximum areas and mean and maximum intensities seem to increase linearly with lifetime; subsequently, this correlation is broken. The only exceptions are the mean area and intensity of THEMIS99 series, which show no evident change with lifetime, but this could originate from inherent differences in the observed granulation. The other plots agree in retrieving an increase of $\sim 20 \%$ of the mean structure area (corresponding to $\sim 30 \%$ in mean cell area), and an increase of $\sim 55 \%$ in the maximum structure area (corresponding to $\sim 65 \%$ in maximum cell area). It is straightforward to derive from this behaviour that large granules have a higher probability of lasting longer than small granules. The maximum area has a steeper dependence on lifetime than average area, denoting larger size fluctuations in larger granules. Such results are in good agreement with the

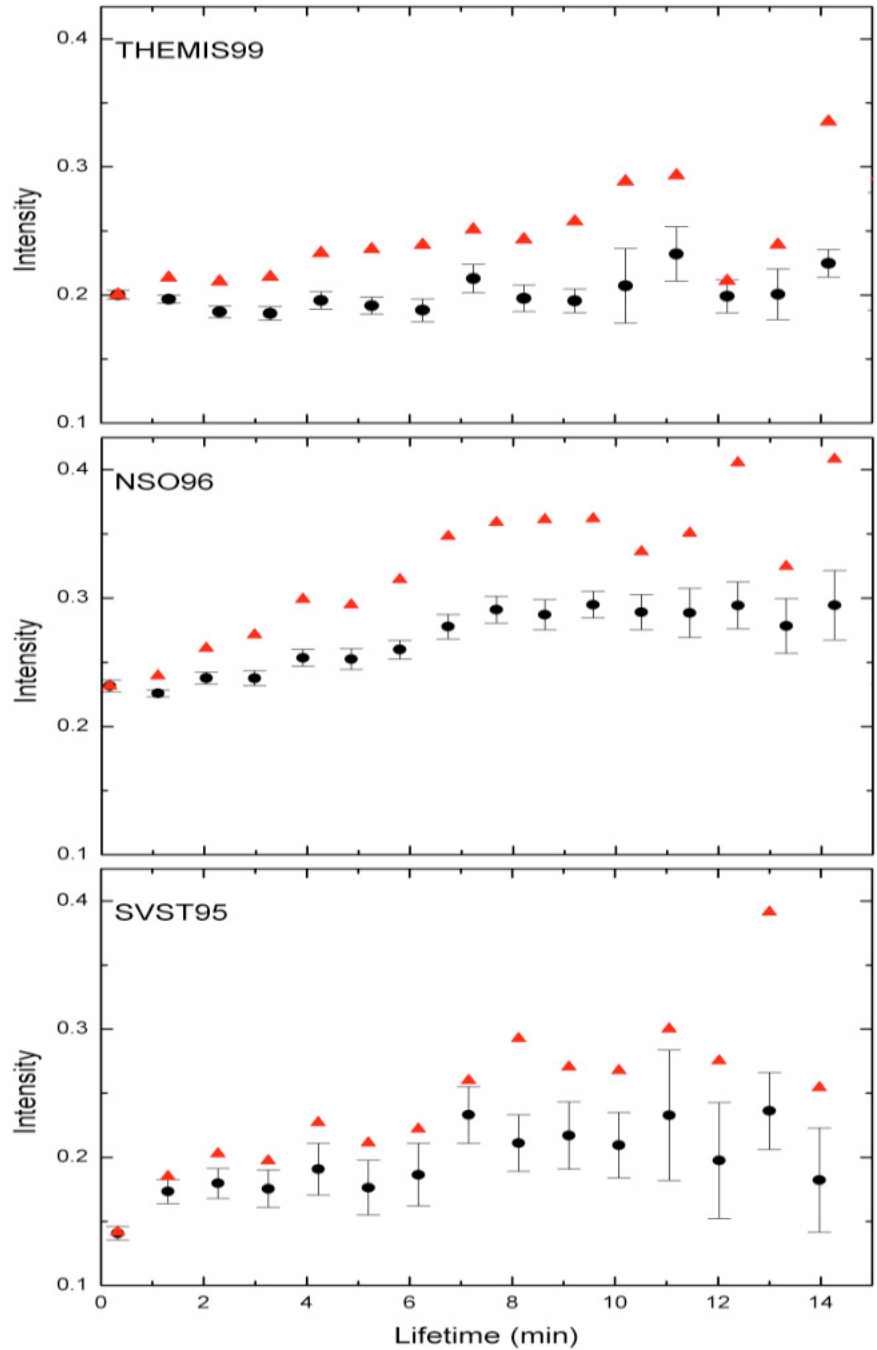

Fig. 6. Plot of granule mean (black filled circles with error bars) and maximum (grey triangles) normalized intensity during lifetime versus their lifetime. Maximum intensity error bars, comparable to mean intensity error bars, are omitted for clarity.

granule simulation of Ploner et al. (1998), which detected a knee in the intensity-lifetime plots exactly at $8 \mathrm{~min}$ of duration. However, we acknowledge that this strict accord might be coincidental. In previous works, similar behaviours have been found also by Title et al. (1989), who found a steep increase in the granular sizes for short lifetimes and almost no variation for very long lifetimes, with a smooth change around $\sim 6 \mathrm{~min}$; Hirzberger et al. (1999a), instead, detected a sort of "kink" at about $12 \mathrm{~min}$ in both area and intensity variations with lifetime. It is comforting to observe that our findings are bracketed by theirs.

\subsection{Horizontal velocity fields}

As reported in Roudier et al. (1999) and Rieutord et al. (2001), the plasma flows can be correctly reconstructed by tracked granules only for spatial scales larger than $2.5 \mathrm{Mm}$ and for temporal scales longer than $30 \mathrm{~min}$, otherwise the derived flow would be spoilt by the granule velocity field. Nevertheless, 
we chose to compute the velocity fields at higher rate and resolution, because we are also interested in the granule proper displacements. The plasma flow can still be obtained by successively averaging the velocity fields, in order to reach the required time and spatial scales. In Fig. 7 we present a pair of horizontal velocity fields (both the sparse array (white arrows) and the interpolated one (black arrows)), separated by 4 min.

Exploding granules, defined as granules that undergo more or less symmetrical fragmentation after a fast expansion phase, and their relationship with mesogranulation are a topic much discussed in recent years. Since its discovery by November et al. (1981), mesogranulation has been analyzed using many different techniques (see Rieutord et al. 2000 for a synopsis of such studies) to reveal its nature. A universal result of these analyses is the detection of some kind of signature in the mesogranulation range of scales, i.e. length scales between $3 \mathrm{Mm}$ and $10 \mathrm{Mm}$ and time scales between $30 \mathrm{~min}$ and $6 \mathrm{~h}$. Recent works of Roudier et al. (2003) and Rast (2003) showed how coherent diverging flows, produced by granule-granule interaction, may generate meso-scale features.

Examining the reconstructed horizontal velocity fields obtained by the TST, we found several roughly ring-shaped structures of divergent flow vectors, corresponding to exploding granules, due to the interaction between the exploder expansion and its neighbours, lasting for several minutes. The pair of images reported here shows an example of such structures.

The interaction of the exploder with its neighbours is highlighted by the pairs of white rings: in the first image the exploder (in the inner circle) has just fragmented and its expansion is now dislocating its neighbours (in the space between the circles). In the second image the TST has already recognized and tracked the five heir fragments (in the inner circle), whose barycentres are the origins of the white arrows and which are still receding from each other; the granules in the space between the circles seem still displaced or at least deflected from the fragments and in the lower-right part a broad intergranular lane has formed. We can therefore define as the typical interaction length for exploders the radius of the outer circle, which is about 4 arcsec. Taking into consideration that a) only very big granules explode, and $b$ ) the greater the granule the longer its duration (cf. 4.3), we can now conclude that these interactions may very well persist several minutes (typically ten-twelve), and may repeat at or very near the same location because, frequently, exploder's fragments undergo repeated fragmentation (Carlier et al. 1969; Roudier et al. 2003).

Even though it cannot be excluded that exploding granules have a tendency to rise near the centre of mesogranular convective cells, whose flow may help them expand, the probable cause of the divergence signal is the exploding granule, as the exploder's fragments are moving with velocities of $\sim 1 \mathrm{~km} \mathrm{~s}^{-1}$ at least and up to $\sim 7 \mathrm{~km} \mathrm{~s}^{-1}$ (Hirzberger et al. $1999 \mathrm{~b}$ and references therein), while the divergence due to mesogranulation is $\sim 1 \mathrm{~km} \mathrm{~s}^{-1}$ at most and peaked around $0.5 \mathrm{~km} \mathrm{~s}^{-1}$ (November \& Simon 1988; Roudier et al. 1998).

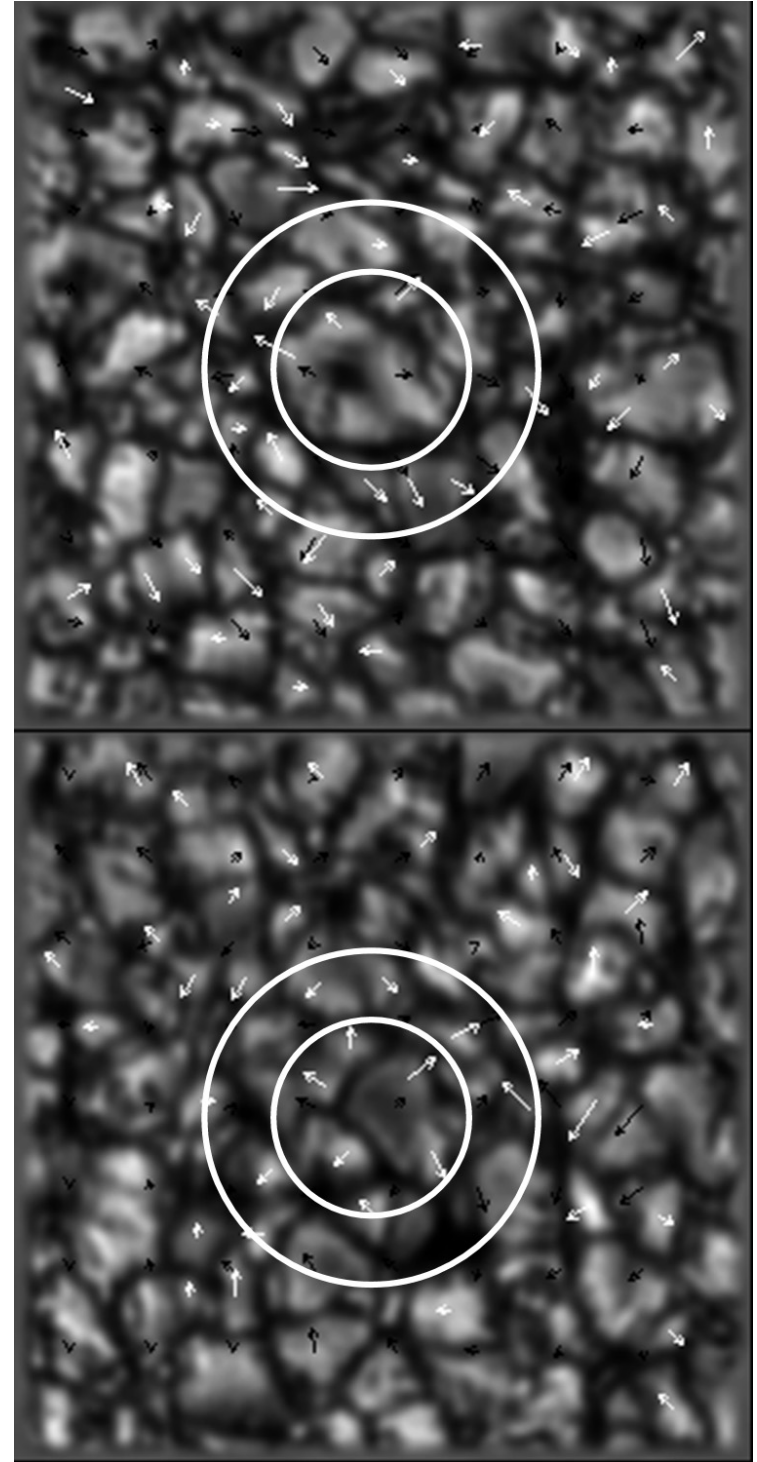

Fig. 7. A pair of original broad-band SVST95 images, with horizontal velocity field superposed. White arrows represents velocity vectors computed from actual granules displacements, black arrows represent the interpolated velocity field. In the centre of the upper image an exploding granule just after fragmentation is visible. The two extracted velocity fields are $4 \mathrm{~min}$ apart. It is evident how the explosion event affects the movement of the nearby granules.

\subsection{Dataset comparison}

We now compare the three datasets. The very good seeing conditions and the effective procedure of re-centring and destretching employed made the SVST95 series the most reliable for the structure tracking and the consequential horizontal flow field reconstruction; however its relatively small FoV limited the statistical significance of the extracted granule characteristics, as can be deduced by the large error bars in Figs. 5 and 6. The NSO96 timeseries, instead, benefits the high statistics offered by a large FoV, while the destretching allows an accurate flow field restoration for the whole timeseries; on the other hand, the TST procedure would have benefited from higher spatial resolution, in order to better resolve the granule structure. Also 
the large FoV of THEMIS99 timeseries guarantees a higher stability in the measure of granule characteristics; however, the IPM acquisition mode required long integration times, thus preventing the removal of some anomalies in the tracking process by destretching or despeckling algorithms and spoiling a few flow field estimations. These tracking problems may also have produced a bias towards shorter lasting granules (as suggested by the lifetime distribution); however, they cannot account for the flatter dependence of granule area and brightness on lifetime duration. Those aspects, instead, could be explained by intrinsic differences in the quiet sun shown in the NSO96 and SVST95 timeseries (acquired near solar minimum) and the THEMIS99 timeseries.

\section{Conclusions}

In this paper, we investigated different aspects of granulation applying a newly developed Two-level Structure Tracking (TST) procedure to three different photospheric broadband image time series.

The TST procedure extracts histories of single structures, by means of automatic segmentation and recognition algorithms, that can be used to derive horizontal velocity fields and granule temporal properties. Applying the TST procedure to the THEMIS99, NSO96 and SVST95 image series, allowed us to perform a statistical analysis of the time dependence of several granule characteristics.

We can recapitulate our results as follow:

To describe the granule lifetime distributions, two class of functions have been used: a single exponential decay law and a stretched exponential decay law. While the first fitted only one part of the histogram, the second matched much closer the whole distribution. Such a behaviour of lifetime distributions implies a dynamical heterogeneity in the low photosphere, as in out-of-equilibrium systems, that prevents the definition of "typical" granule duration.

The time-averaged size distributions of granules and of granular cells show a small scale cut-off due to the resolution limit of observations, but also a large scale cut-off, implying that the time-averaged cell diameter maximum value is $\sim 2.0 \mathrm{Mm}$.

The size-lifetime tables show a well defined trend of large granules surviving longer. The $\sim 8 \mathrm{~min}$ knee in this plot is in agreement with previous observational (Title et al. 1989; Hirberger et al. 1999a, although they found this knee around 6 and at $\sim 12$ min respectively) and theoretical (Ploner et al. 1998) works.

The analysis of the horizontal velocity field near an exploding granule has been of particular interest. Since the work of November et al. (1981), several authors have tried to correlate granular properties with their position in mesogranular cells (see for example Brandt et al. 1991). Recently Roudier et al. (2003) and Rast (2003) have suggested that instead granule properties could be the cause of mesogranular scale divergence signals. We believe that our findings are of considerable significance in the debate, as they show how an exploding granule may create a divergence signal on the mesogranular scale by displacing its neighbours.
Acknowledgements. The author is indebted to F. Berrilli, J. A. Bonet and G. Cauzzi for kindly allowing him to use their three datasets. Particular thanks to F. Berrilli, for advice and assistance during the developing of the author's degree thesis, from which a great part of this research derives. The author is also grateful to G. Consolini and A. Florio for fruitful discussions and to J. A. Bonet, C. Briand for reading the manuscript and for providing valuable comments. The THEMIS is operated on the island of Tenerife by CNRS and CNR in the Spanish Observatorio del Teide of the Instituto de Astrofísica de Canarias. The Dunn Solar Telescope is operated by the Association of Universities for Research in Astronomy, Inc. (AURA) at the National Solar Observatory in Sacramento Peak (New Mexico). The Swedish Vacuum Solar Telescope is operated on the island of La Palma by the Royal Swedish Academy of Science in the Spanish Observatorio del Roque de Los Muchachos of the Instituto de Astrofisica de Canarias. Special thanks to the THEMIS crew and, in particular, to G. Ceppatelli, C. Briand and G. Mainella. The author would like to acknowledge the referee R. Muller's helpful and valuable comments. This work was partially supported by the Italian National Research Council (CNR) grant Agenzia2000 CNRC0084C4 and by the MIUR grant COFINO2.

\section{References}

Alfeld, P. 1989, in Mathematical Methods in Computer Aided Geometric Design, ed. T. Lyche, \& L. L. Schumaker (Academic Press), 1

Alissandrakis, C. E., Dialetis, D., \& Tsiropoula, G. 1987, A\&A, 174, 275

Berrilli, F. 2003, MmSAIt, 74, 576

Berrilli, F., Florio, A., \& Ermolli, I. 1998, Sol. Phys., 180, 29

Berrilli, F., Consolini, G., Florio, A., \& Pietropaolo, G. 2001, MmSAIt, 72, 669

Berrilli, F., Consolini, G., Pietropaolo, E., et al. 2002, A\&A, 381, 253

Bovelet, B., \& Wiehr, E. 2001, Sol. Phys., 201, 13

Brandt, P. N., Ferguson, S. H., Scharmer, G. B., et al. 1991, A\&A, 241, 219

Carlier, A., Chauveau, F., Hugon, M., \& Rösch, J. 1969, C. R. Acad. Sci. Paris B, 266, 199

Cauzzi, G., Consolini, G., Berrilli, F., et al. 1998, MmSAIt, 69, 647

Del Moro, D. 2001, Degree Thesis at Physics Dept., Rome Tor Vergata University

Gadun, A. S., Hanslmeier, A., \& Pikalov, R. N., et al. 2000, A\&AS, 146, 267

Hirzberger, J., Vázquez, M., Bonet, J. A., Hanslmeier, A., \& Sobotka, M. 1997, ApJ, 480, 406

Hirzberger, J., Bonet, J. A., Vázquez, M., \& Hanslmeier, A. 1999a, ApJ, 515, 441

Hirzberger, J., Bonet, J. A., Vázquez, M., \& Hanslmeier, A. 1999b, ApJ, 527, 405

Koschinsky, M., Krieg, J., Kneer, F., Ritter, C., \& Wunnenberg, M. 1999, High Resolution Solar Physics: Theory, Observations, and Techniques, ed. Rimmele, Balasubramaniam, \& Radick, ASP Conf. Ser., 183, 349

Lancaster, P., \& Salkauskas, K. 1986, Curve and Surface Fitting: An Introduction (Academic Press)

Nordlund, ̊.. 1984, A\&A, 107, 1

November, L. J., \& Simon, G. W. 1988, ApJ, 333, 427

November, L. J., Toomre, J., Gebbie, K. B., \& Simon, G. W. 1981, ApJ, 245, L123 
Ploner, S. R. O., Solanki, S. K., Gadun, A. S., \& Hanslmeier, A. 1998, Space Sci. Rev., 85, 261

Rast, M. P. 2003, ApJ, 597, 1200

Rieutord, M., Roudier, Th., Malherbe, J. M., \& Rincon, F. 2000, A\&A, 357,1063

Rieutord, M., Roudier, Th., Ludwig, H. G., Nordlund, ̊., \& Stein, R. 2001, A\&A, 377, L14

Roudier, Th., \& Muller, R. 1987, Sol. Phys., 107, 11

Roudier, Th., Malherbe, J. M., Vigneau, J., \& Pfeiffer, B. 1998, A\&A, 330, 1136

Roudier, Th., Rieutord, M., Malherbe, J. M., \& Vigneau, J. 1999, A\&A, 349, 301

Roudier, Th., Ligniéres, F., Rieutord, M., Brandt, P. N., \& Malherbe, J. M. 2003, A\&A, 409, 299
Schrijver, C. J., Hagenaar, H. J., \& Title, A. M. 1997, ApJ, 475, 328

Sobotka, M., Vázquez, M., Bonet, J. A., Hanslmeier, A., \& Hirzberger, J. 1999, ApJ, 511, 436

Spruit, H. C., Nordlund, Å., \& Title, A. 1990, ARA\&A, 28, 263

Steffen, M., Ludwig, H. G., \& Kruss, A. 1989, A\&A, 213, 371

Stein, R. F., \& Nordlund, ̊.. 1998, ApJ, 499, 914

Strous, L. 1995, Feature tracking: deriving horizontal motion and more, in Proc. of 4th SOHO Workshop: Helioseismology, ESA SP-376, 219

Title, A. M., Tarbell, T. D., Topka, K. P., Ferguson, S. H., Shine, R. A., \& SOUP Team 1989, ApJ, 336, 475

Williams, G., \& Watts, D. C. 1970, Trans. Faraday Doc., 66, 80 